\title{
STUDIES ON TRANSMEMBRANE ACTION POTENTIALS AND MECHANICAL RESPONSES OF THE VENAE CAVAE AND ATRIA OF THE RABBIT
}

\author{
Makoto Arita, Kazuyuki Saeki, Mitsuo Tanoue, Morio Ito, \\ Takasi YanAGA AND Hiroto MashibA \\ First Department of Internal Medicine (Director: Prof. K. Yamaoka) \\ Faculty of Medicine, Kyushu University, Fukuoka, Japan
}

Since the pulsation of the vena cava was first noticed in the dog by Wallaenus in 1660 (FRANKLIN, 1937), the existence of rhythmical contractions of the venae cavae proximal to the heart in various animals has been reported (Brunton \& FAyrer, 1876 ; Engelmann, 1897 ; Ochiai, 1931 ; Franklin, 1937 ; Senba, 1941 ; Nishimoto, 1951).

Recently, MASHIBA et al. (1963) demonstrated that the impulse originating in the sinus node spread not only to the atrial muscle but also to the vena caval muscle, and nominated this phenomenon "sino-caval conduction."

From the viewpoint of embryology (AREY, 1965) and hemodynamics (GARUSI, 1965), propagated excitation and contraction in the vena cava are important problems. Up to the present time, however, no observation has been reported about the relationship between excitation and contraction in the vena cava and their propagation from the heart.

The purpose of this paper is to elucidate the electrical activities and mechanical responses of the vena caval muscle, in comparison with those of the atrial muscle, by the aid of intracellular microelectrodes and a strain gauge transducer.

\section{METHODS}

After an intraperitoneal injection of pentobarbital sodium, an adult rabbit was thoracotomized under artificial respiration. The heart was excised with three venae cavae cut $2 \sim 3 \mathrm{~cm}$ from each cardiac orifice, and a large part of both ventricles was cut away.

The experiments were performed by using two different types of preparation. They are: (1) The atrio-caval preparation, composed of three venae cavae and both atria (FIG. 1), i. e., the left superior vena cava (LSVC), the right superior vena cava (RSVC), the inferior vena cava (IVC), the left atrium (LA) and the right atrium

Received for publication January 29, 1966

有田 真, 佐伯和之, 田上三雄, 伊東盛夫, 矢永尚士, 真柴裕人 
(RA). This preparation commonly showed automatic rhythmical contractions under the sinus rhythm as a whole. (2) The strip preparation, dissected from various parts of the atrio-caval preparation, which are named LSVC-, RSVC-, IVC-, RA-, and LA-strip respectively. In this dissection, the atrial strips were cut so that the most prominent fiber bundles would run parallel, and the vena caval strips were cut as a longitudinal tubular segment. Their size was about $20 \sim 30 \mathrm{~mm}$ in length, $2 \sim 3 \mathrm{~mm}$ in width and dess than $1 \mathrm{~mm}$ in thickness.

The above-illustrated atrio-caval or strip preparation was mounted horizontally in a muscle bath irrigated with oxygenated Tyrode solution at the constant temperature of $35^{\circ} \mathrm{C}$ so that the dorsal side faced upward in in situ length.

When necessary, the preparation was driven by isolated rectangular electrical pulses of $5 \mathrm{msec}$ in duration and $5 \sim 15$ volts in strength through a stimulating elec. trode attached to its surface.

Simultaneous recordings of transmembrane action potentials and isometric tension of the muscle were accomplished by a flexibly mounted microelectrode and a UL-gauge (Shinkoh Tsushin Co.), the latter of which was connected to several portions of the atrio-caval preparation or the center of the strip preparation with the aid of a silk thread and serre-fine at right angles to the surface. Penetrations were made through the external surface at a point of a few millimeters from the site connected to the strain gauge.

The potential and tension changes were amplified and displayed on a dual beam cathode-ray oscilloscope and/or an ink-writing oscillograph.

\section{RESULTS}

1) Spread of contraction. FIG. 1 is a schema of the atrio-caval preparation illustrating time delays in milliseconds between the upstroke of action potentials recorded at one point $(x)$ of the crista terminals and the onset of corresponding contractions recorded from various parts. This figure indicates that a contraction-wave originating near the sinus node spreads at first to the crista terminalis, and then to the more distal parts of the LSVC and RSVC, as well as the both atria.

The mode of propagation of the contraction was nearly coincident with that of electrical activity (ITO et al.: to be published). Hence, it is evident that the direction of the spread of contraction in the superior venae cavae (SVC) is going against the blood stream.

From the hemodynamic aspect, it seems interesting that the crista terminalis, the orifice of the SVC, and their neighbouring parts were found to contract $20 \sim 30 \mathrm{msec}$ earlier than the RA. This problem will be discussed later.

2) Simultaneous recordings of action potential and contraction. Spontaneous contractions of the SVC in the atrio-caval preparation could be recorded, synchronized with action potential discharge (FIG. 2, A and B) similar to that of the both atria (FIG. 2, C and D). In the strip preparations, however, the SVC were usually destitute of the spontaneous activities except a few cases of the 


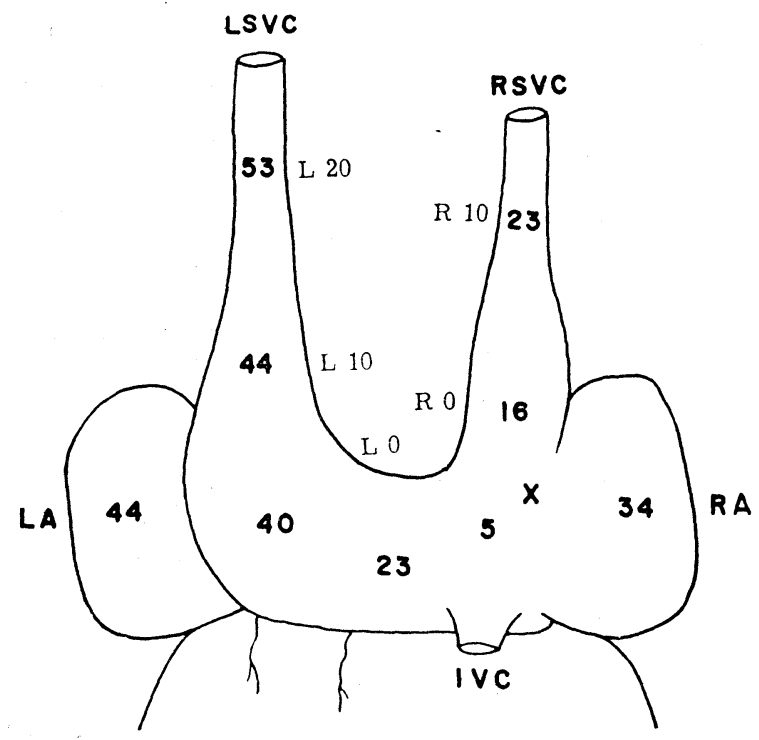

FIG. 1. A schema showing the mode of spread of contraction in the atrio-caval preparation, the numbers represent time delays in milliseconds from the upstroke of an action potential at the one point $(x)$ of the crista terminalis to the onset of the corresponding contraction in various parts. Other designations are as follows: LSVC, the left superior vena cava; RSVC, the right superior vena cava; IVC, the inferior vena cava; LA, the left atrium; RA, the right atrium; $\mathrm{L}_{0}$, orifice of the LSVC; $\mathrm{L}_{10}, 10 \mathrm{~mm}$ distal from $\mathrm{L}_{0} ; \mathrm{L}_{20}, 20 \mathrm{~mm}$ distal from $\mathrm{L}_{0} ; \mathrm{R}_{0}$, orifice of the RSVC ; $R_{10}, 10 \mathrm{~mm}$ distal from $\mathrm{R}_{0}$.

LSVC-strip as shown in FIG. 2, I and J. But the electrical stimulation have easily produced action potentials and contractions in the strips of the SVC (FIG. 2, E and F) as well as the atria (FIG. 2, G and H).

On the contrary, neither action potential nor contraction could be recorded from the IVC in the atrio-caval preparation as well as the IVC-strip even under the electrical stimulation.

In general, the manner of contraction of the SVC was so-called a 'twitch" educed by a single action potential.

3) Unusual contraction. (a) When the atrio-caval preparation showing spontaneous activity was concurrently applied with electrical stimuli of considerably lower frequency, it showed sometimes transient bursts of rapid action potentials followed by rapid contractions of the same frequency in both the LSVC and LA (FIG. 3, A-1 and B-1). This phenomenon took place almost only when a stimulus was accidentally synchronized with a particular phase of repolarization of the preceding action potential as indicated in FIG. 3, A-3 and B-3. This particular phase in relative refractory period in the atrio-caval preparation. 

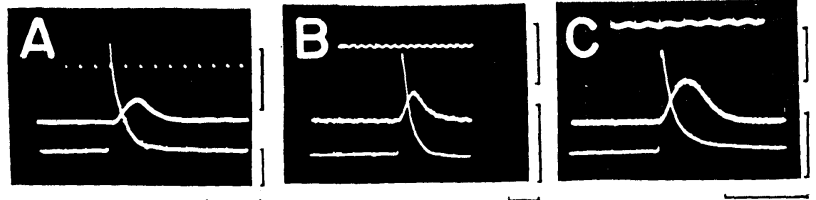

-
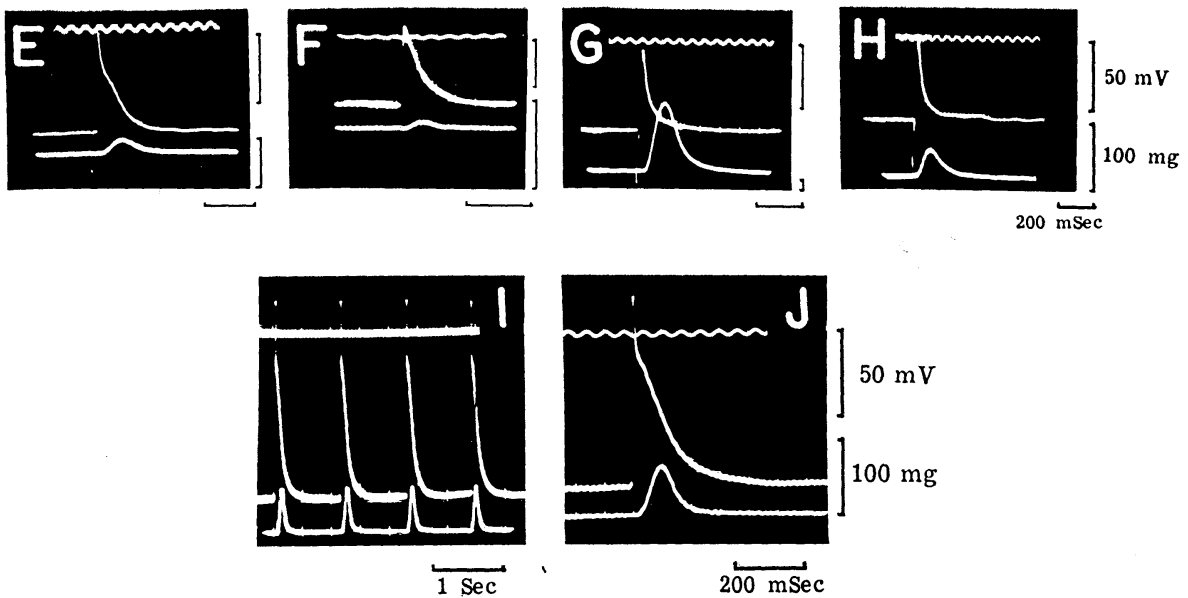

$50 \mathrm{mV}$

$100 \mathrm{mg}$

FIG. 2. Simultaneous records of action potential and contraction. A, B, C and $\mathrm{D}$ are spontaneous activities of the LSVC, RSVC, LA and RA in the atrio-caval preparation respectively. E, F, G and $\mathrm{H}$ are electrically driven activities of the strips of the LSVC, RSVC, LA and RA respectively. I and J are records obtained from a strip of the LSVC showing spontaneous contractions, which were seemed considerably of rare occurrence. In each record, upper beam shows $20 \mathrm{cps}$ of time calibration; middle and lower beam, membrane potential and contractile tension.

seems to correspond to the "vulnerable period" (BROOKs et al., 1955): it is stated that if a stimulus of appropriate strength has been applied at these particular intervals of cycle of the atrium or ventricle, single extrasystoles, multiple responses, or flutter or fibrillation of brief duration occur.

(b) FIG. 3, A-2 represents an incomplete tetanus like contraction associated with contractile summation, which was occasionally recorded from the LSVC in the atrio-caval preparation. In contrast, the recording of similar phenomenon from the LA resulted in failure.

(c) At the increased stimuls frequency above $7 \sim 8 \mathrm{cps}$, the dissociation of excitation-contraction coupling (E-C coupling) accompanied by the electrical conduction block were sometimes occurred in the LSVC-strip as shown in FIG. 4, viz., some action potentials failed to elicit the effective contraction. But the dissociation of $\mathrm{E}-\mathrm{C}$ coupling seemed to be of extremely rare occurrence in the case of the LA-strip. 


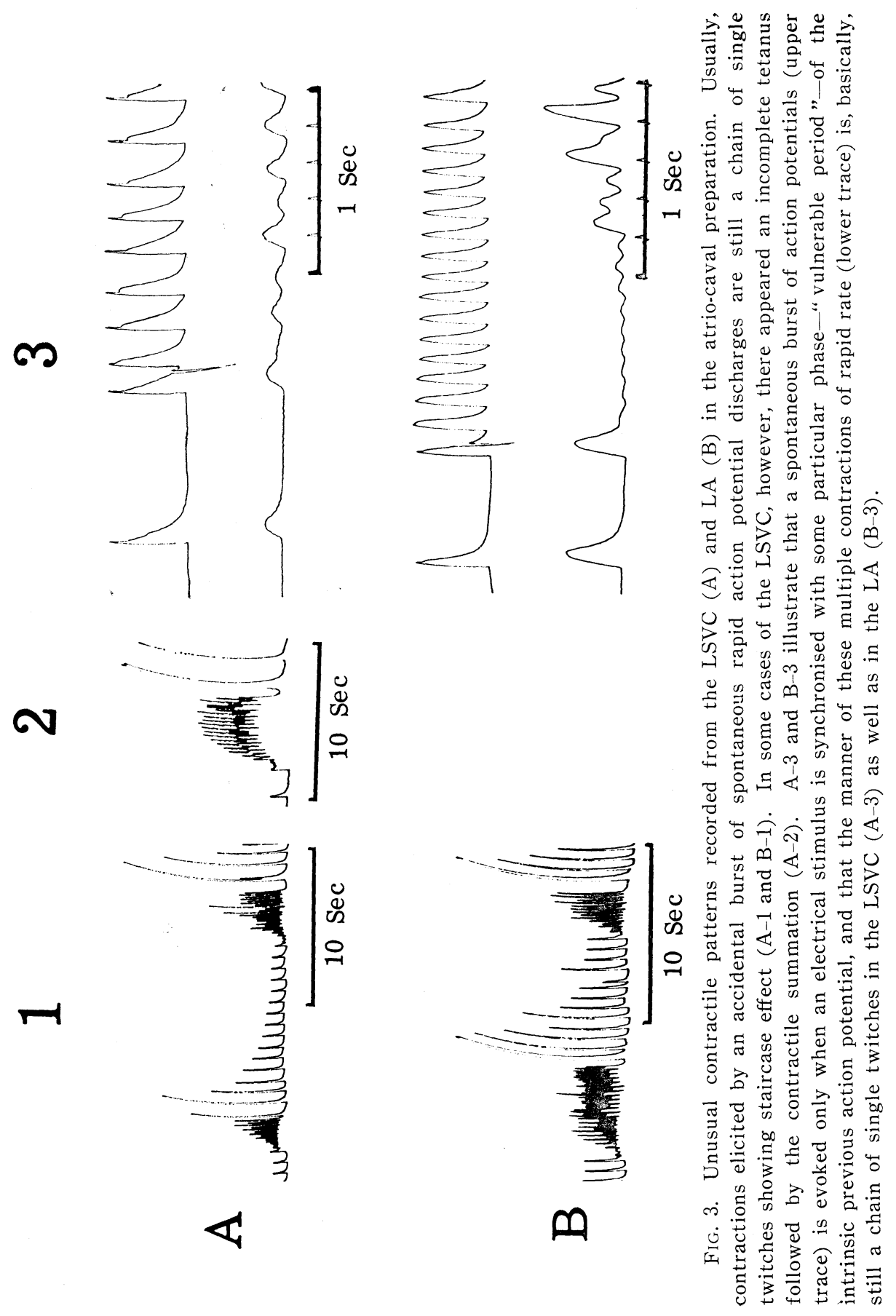




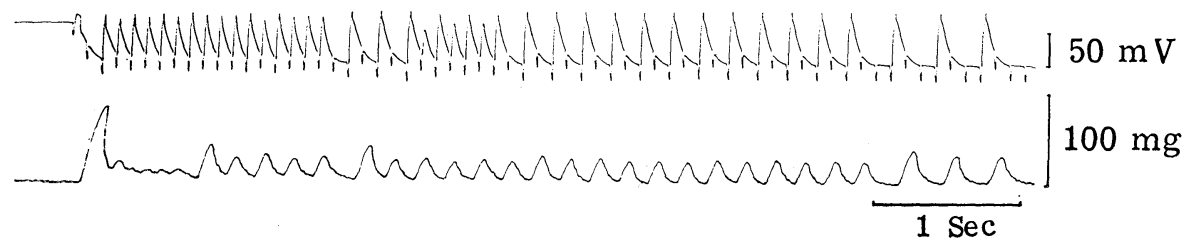

FIG. 4. Dissociation of E-C coupling which was observed in the strip of the LSVC driven electrically at a rate of $1.0 \mathrm{cps}$. Upper trace is action potentials, and lower, contractions. Initial straight line in the upper trace indicates 0 -volt level.

4) Staircase phenomenon. As indicated in FIG. 5, the LSVC-strip (A) as well as the LA-strip (B) exhibited a striking staircase phenomenon when the stimulus frequency was abruptly shifted from a lower to a higher value or vice versa. Their development is essentially identical with the observations reported by other investigators using various cardiac muscle preparations (Hajdu, 1935 ; Cattel \& Gold, 1941 ; Vaughan Williams, 1959).

A

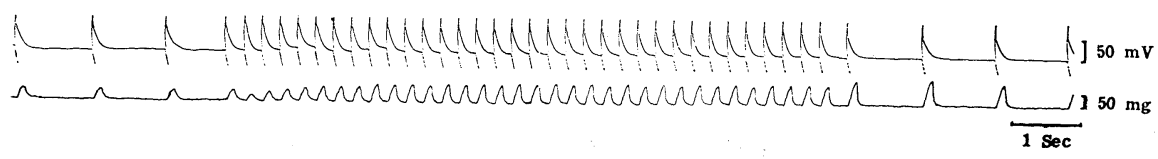

B

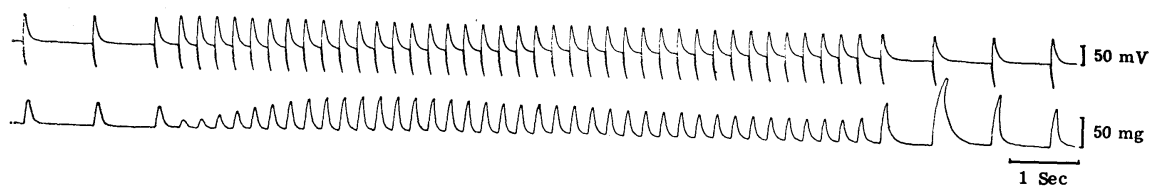

Fig. 5. Normal and reverse staircase phenomena recorded from the strips of the LSVC (A) and LA (B). In both A and B, stimulus frequency was raised abruptly from $1 \mathrm{cps}$ to $4 \mathrm{cps}$, and several seconds later, turned back to $1 \mathrm{cps}$. Upper trace in each record is action potentials, and lower trace, contractions. Further explanation is in text.

It showed a several-fold increase in the contractile force when the rate of stimulus was changed from a slow $(1 \mathrm{cps})$ to a fast $(4 \mathrm{cps})$ one (normal staircase), and a decrease when the rate was changed back in the reverse direction (reverse staircase).

These changes developed gradually: the first few contractions in the transition from a slow to a rapid rate were smaller than the previous contractions ; conversely, the first few contractions in the transition from a rapid to a slow rate were larger than the previous rapid beats and subsequent slow beats.

In the normal staircase effect set up by stimulation at a rapid rate of 4 cps or more, there appeared two opposing processes influencing contractility. One is facilitatory, in which each contraction results in a greater tension of the next contraction. The other is inhibitory, in which contractions show 
gradual decrease. As shown in FIG. 5, when the frequency was shifted abruptly from $1 \mathrm{cps}$ to $4 \mathrm{cps}$, the LA-strip (B) was inclined to reach a peak tension more rapidly (facilitatory process) and veer to a gradual decrease more easily (inhibitory process) than the LSVC-strip (A).

5) Relation between stimulus frequency and contraction strength. The graphs representing the frequency-tension relationship of various cardiac muscles seem to be considerably different from one another according to the individual animals used (VANE, 1957; Hoffman \& Kelly, 1959; VAughan Williams, 1959). Hence, the influence of stimulus frequency on contractility was investigated comparing the LSVC with the LA.

As shown by the solid curves in FIG. 6, both the LSVC and the LA-strips showed large contraction at a very low frequency. With increasing frequency, there appeared, at first, very small contraction which was provisionally named ' infinitesimal phase' in contractile force $(\alpha$ and $\beta$ in FIG. 6). Thereafter, the

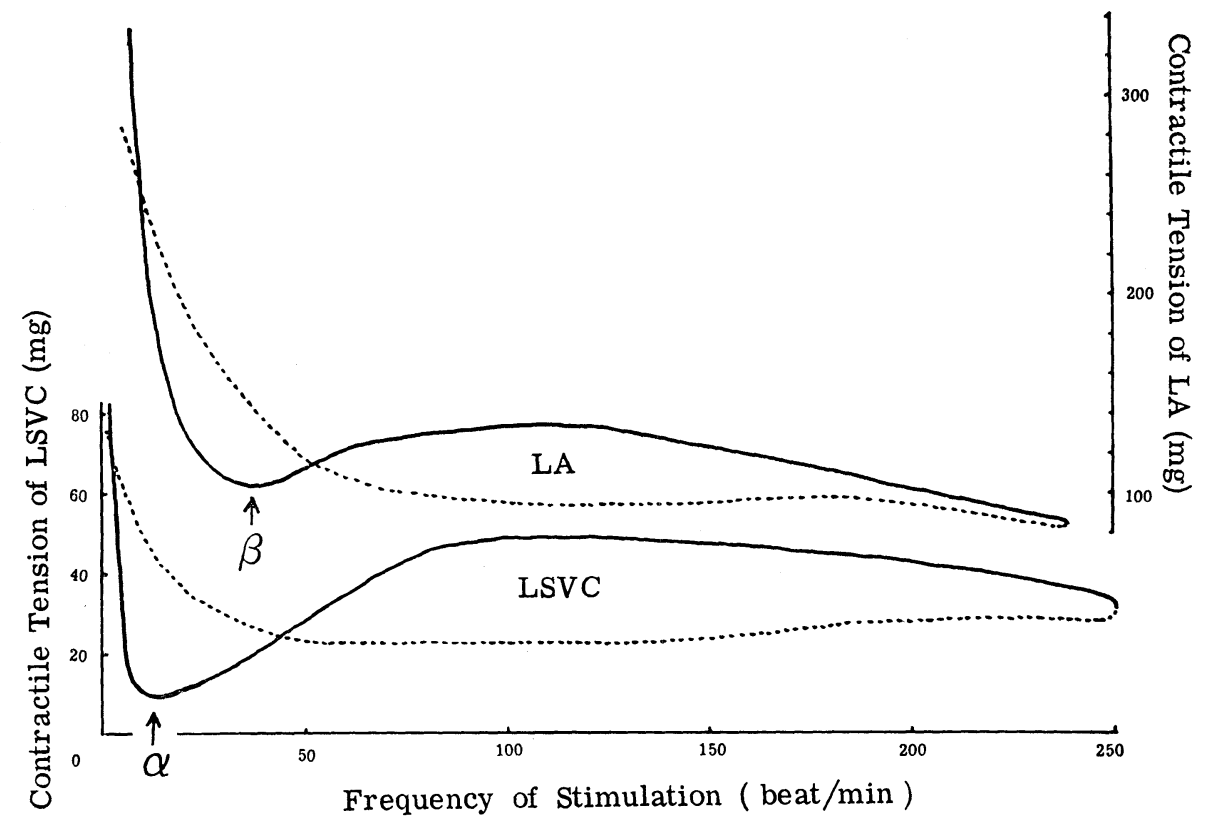

FIG. 6. Relationships between contractile force and stimulus frequency in the typical case of a strip of the LSVC (lower graph) and the LA (upper graph). In each graph, the solid line refers to the case in which frequency was changed from the lower to the higher, and the dotted line, from the higher to the lower. Measurements of contractile tension were carried out when the contractions reached nearly the steady state at every frequency. Alpha and beta represent frequencies corresponding to 'infinitesimal phase' of the contraction strength in the LSVC and LA respectively. The mean values calculated from each 11 cases are as follows: $\alpha=20 \pm 2.7 \mathrm{cps} ; \beta=43 \pm 3.8 \mathrm{cps}$. 
contraction began to increase gradually and reached a maximum followed by a decline at still higher frequencies.

When the frequency was turned back gradually from higher to lower values, the course of contractile force (dotted curves in FIG. 6) did not trace the former one (solid curves in FIG. 6). In this case, however, the course was fairly simple, i. e., the lower the frequency, the larger was the contraction. These findings indicate the existence of a hysteresis phenomenon in contractility of the LSVC as well as the LA.

On the other hand, it was found that the frequency corresponding to the 'infinitesimal phase' of the $\operatorname{LSVC}(\alpha$ in FIG. 6) was usually lower than that of the LA ( $\beta$ in FIG. 6 ) : namely, $\alpha=20 \pm 2.7 \mathrm{cps} ; \beta=43 \pm 3.8 \mathrm{cps}$ respectively.

The frequency producing maximum contractions could not be determined clearly because of their large variance in both the LSVC and LA.

6) Rest contraction. Rest contraction, which was a transient increase in the contractile force elicited by the introduction of a rest interval in the driving stimulus, also occurred in both the LSVC and LA-strip. This contraction was basically identical with the results by other investigators using other types heart muscles (Rosin \& Farah, 1955 ; Hoffman \& Kelly, 1959 ; Sleator et

1
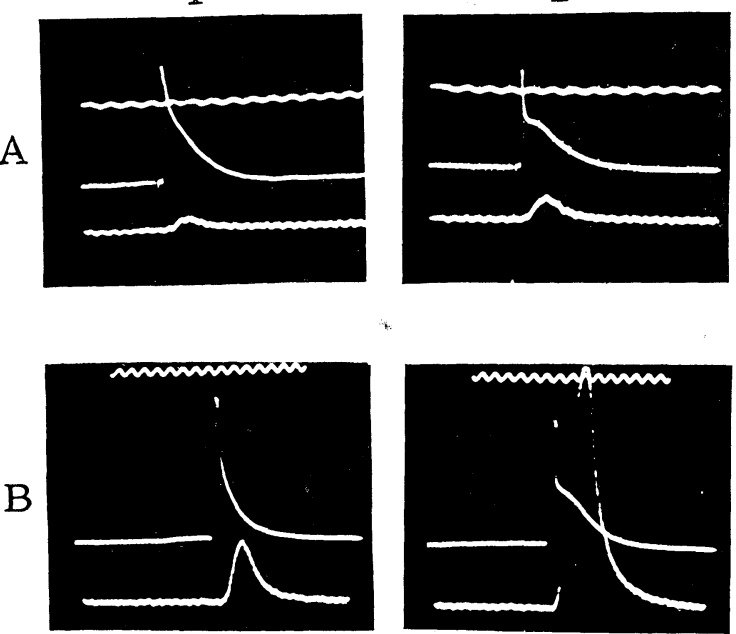

3
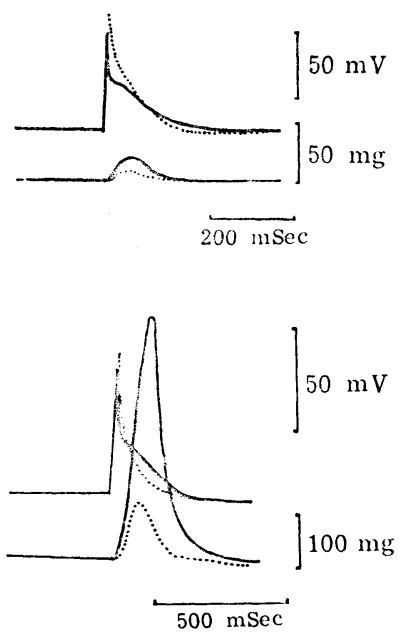

FIG. 7. Relationships between the action potential configuration and the magnitude of contraction at the first rest contraction, recorded from the strips of the LSVC (A) and LA (B). (1): The last beat at the basal rate (1 cps) as a control. (2): The first rest contraction representing marked increase of contractile force associated with an action potential of the shorter initial portion and more prolonged tailing off. Stimulation was ceased during $10 \mathrm{sec}$ between (1) and (2). (3) : Superimposition of (1)-dotted line and (2)-solid line. 
al., 1964).

In both the LSVC and LA-strips, cessation of the basal stimuli of $1 \mathrm{cps}$ for a short time resulted in supernormal contraction (rest contraction) when stimulation was again instituted (FIG. 7). The first rest contraction, especially, was of remarkably greater amplitude (FIG. 7, A-2 and B-2) than the basal contraction (FIG. 7, A-1 and B-1). Moreover, the degree of potentiation in the first rest contraction was variable as a function of the length of the interval of the preceding rest. A concomitant study of this phenomenon was carried out in the LSVC and LA-strips respectively.

As indicated in FIG. 8, the first rest contraction in each strip reached the maximum after a rest of $60 \sim 200 \mathrm{sec}$. After the quiescent period of $1000 \mathrm{sec}$, however, the potentiation of the first rest contraction in the LSVC (solid line) had completely disappeared. In contrast, the first rest contraction after the same quiescence still remained at a highly potentiated state in the LA (dotted line).

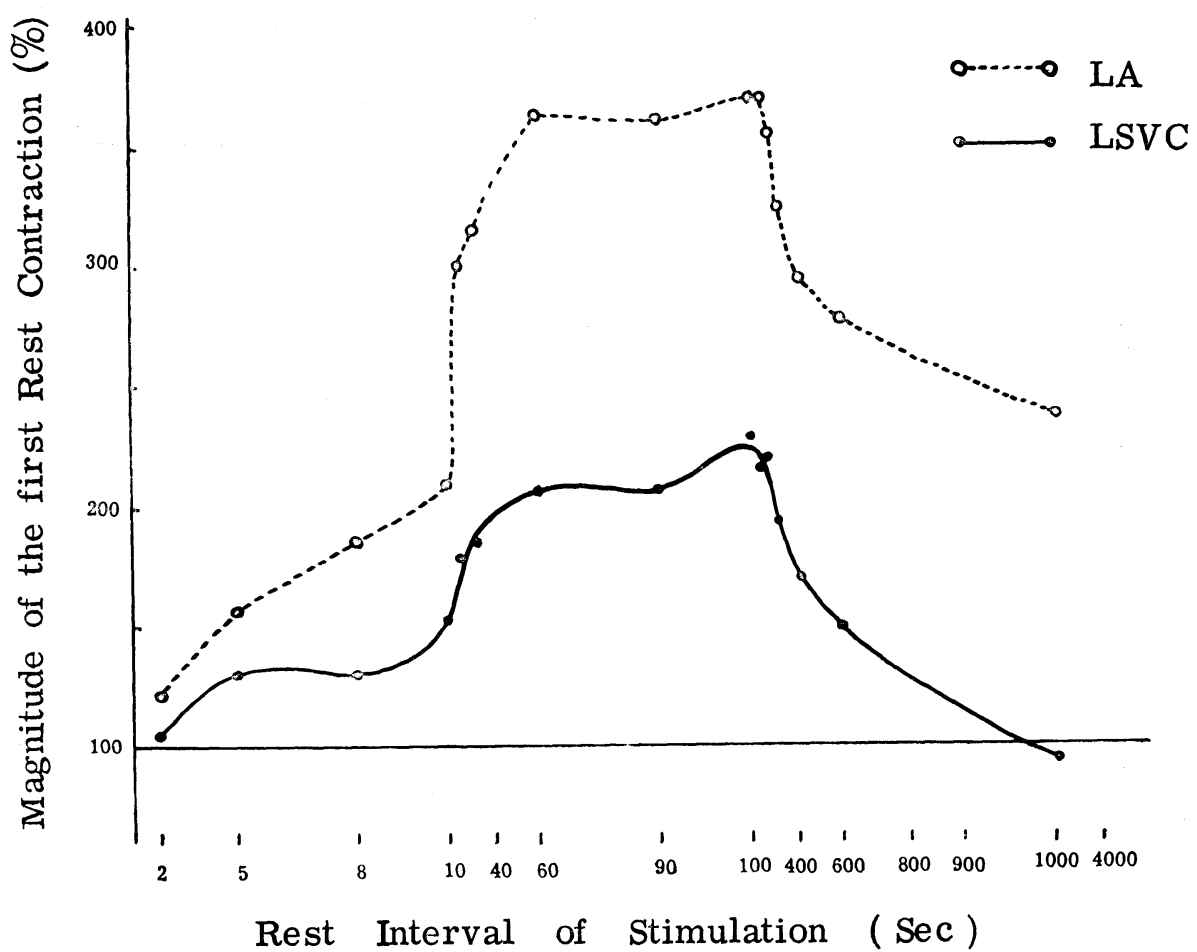

FIG. 8. Comparison of the effects of the rest on the magnitude of contraction in the strips of the LSVC (solid line) and the LA (dotted line). Abscissa: Time in seconds, during which stimuli were ceased. Ordinate: Magnitude of the first rest contraction educed by a stimulus instituted again after a temporary rest (abscissa), which is expressed as percentage of the magnitude of steady state contraction at the basal rate (1 cps). 
The relationship between the shape of action potential and the magnitude of rest contraction is illustrated in FIG. 7, A-3 and B-3. It is evident that the large contraction after a temporary rest $(10 \mathrm{sec})$ is associated with a shortening of the initial portion and a lengthening of the terminal portion of repolarization and a little decrease in the height of an action potential in both the LSVC and LA.

7) Irregular contraction. When electrical stimuli at a rate considerably slower than the intrinsic sinus rate were concurrently applied to the atrio-caval preparation with spontaneous activity, a remarkable arrhythmia occurred in the SVC and atria. This type of arrhythmia is represented in FIG. 9. That is, the magnitude of contractions was drastically variable according to the change in the preceding cycle length and/or the configuration of the corresponding action potential.
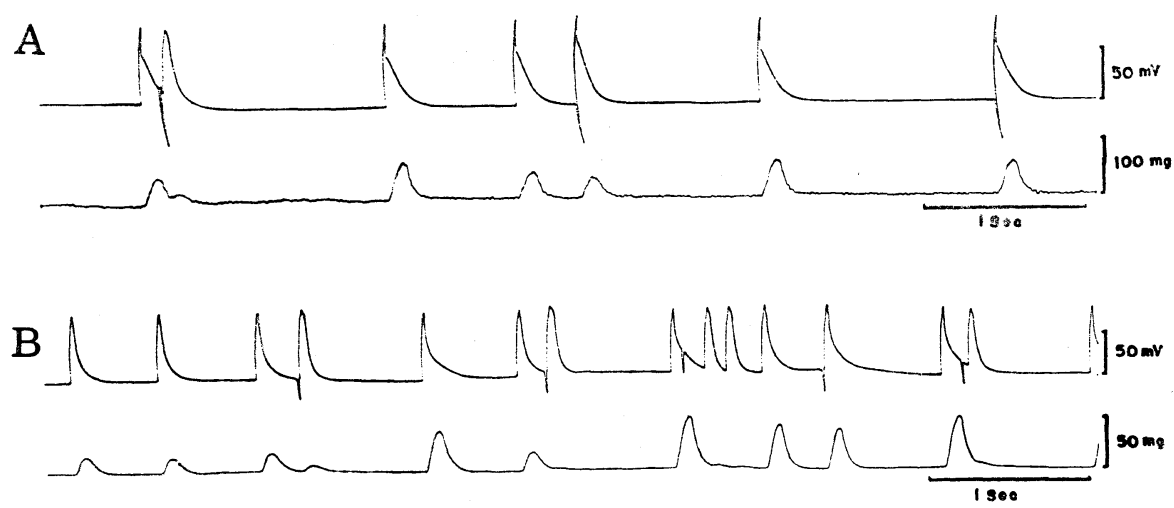

FIG. 9. Irregular contractions of the atrio-caval preparation produced by the concurrent application of electrical stimuli to the preparation with intrinsic (sinus rhythm) spontaneous activity. A : LSVC, automaticity of $0.7 \mathrm{cps}+$ stimulus of 0.4 cps. B: LA, automaticity of $1.7 \mathrm{cps}+$ stimulus of $1.1 \mathrm{cps}$. In both $\mathrm{A}$ and $\mathrm{B}$, the upper trace is action potentials and lower, contractions.

Concerning the relation between the cycle length and the contraction strength, in the terminology illustrated in FIG. 10, C, the magnitude of an arbitrary contraction ' $Z$ ' had no particular relationship to the preceding cycle length ' $y$ ' nor the cycle length ' $x$ ' prior to ' $y$ '. On the other hand, as shown in FIG. 10, $A$ and $B$, there existed a highly positive correlation between ' $z$ ' (ordinate) and ' $y / x$ ' (abscissa) in both of the LSVC and LA: in the LSVC, $\mathrm{r}=0.85(\mathrm{p}<0.001)$; in the LA, $\mathrm{r}=0.98(\mathrm{p}<0.001)$.

Concerning the relation between the action potential configuration and the contraction strength, the height and the shape of an action potential educed by a stimulus during relative refractory period or at progressively later phases of the recovery process, were seen to vary a great deal due to a length of 

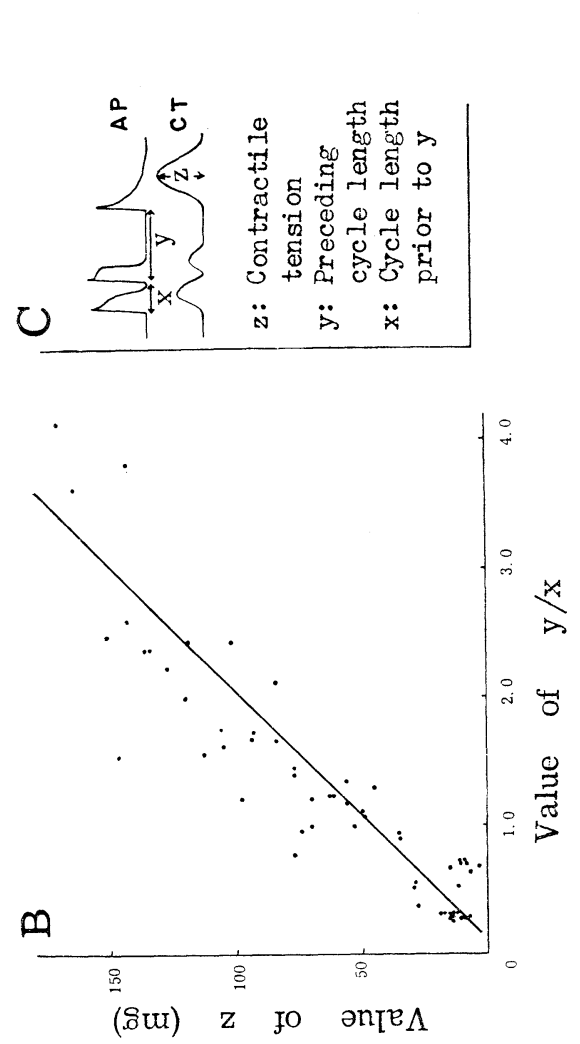

तี $\ddot{\sim}$

它苟

范苛

ฐ 0.

पा 18 है

我

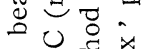

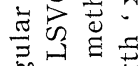

की

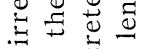

荡零

品

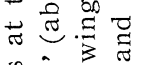

秚

कo $>$ क

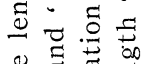

$\div$ ส

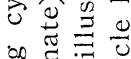

$\infty .=$

过苛

类 i

च

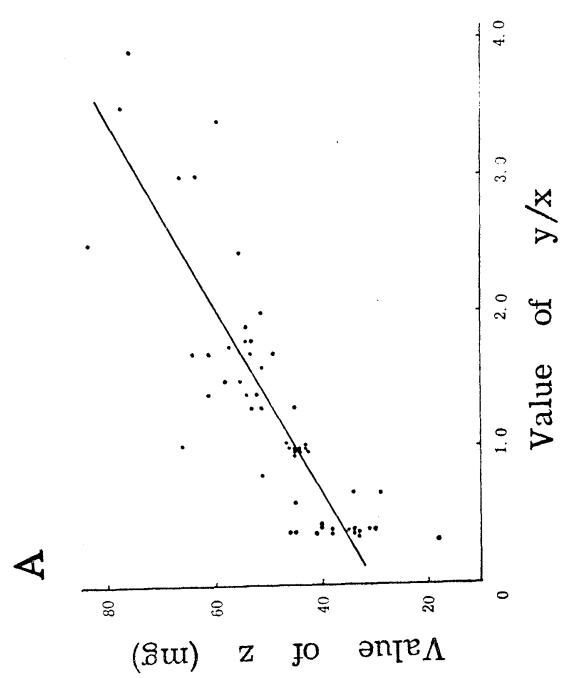

๘ $\ddot{0}$

证.

홍

证 $V$

番

苞茯

a 00

造志

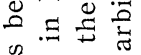

ปี

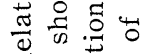

น

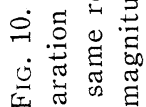

岂芯 
the interval from the onset of previous action potential to the stimulus. Accordingly, these changes in the action potential configuration also seemed to have intimate relation with changes in contractile force (FIG. 9).

Confirming this presumption, in FIG. 11, the magnitude of contraction (abscissa) was plotted against the action potential duration (ordinate) in the same arrhythmia as shown in FIG. 9. In both the LSVC and LA, the longer the $85 \%$-duration and the shorter the 35\%-duration, the stronger was contraction; in contrast, it must be emphasized that $60 \%$-duration was almost
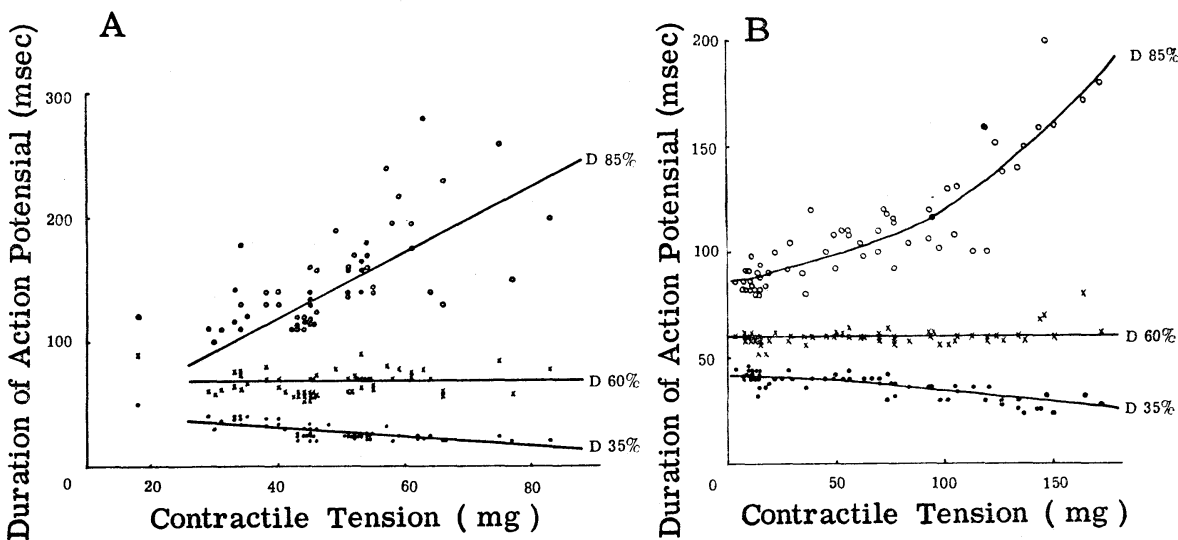

FIG. 11. Relations between the duration of an action potentials (ordinate) and the magnitude of corresponding contractions (abscissa) at the same irregular beats as shown in FIG. 9. A: LSVC. B: LA. D-35\% (O), D-60\% (x) and D-85\% (O) represent the durations of an action potential at the level of $35 \%, 60 \%$ and $85 \%$ in height measured from the top of the action potential respectively.

A
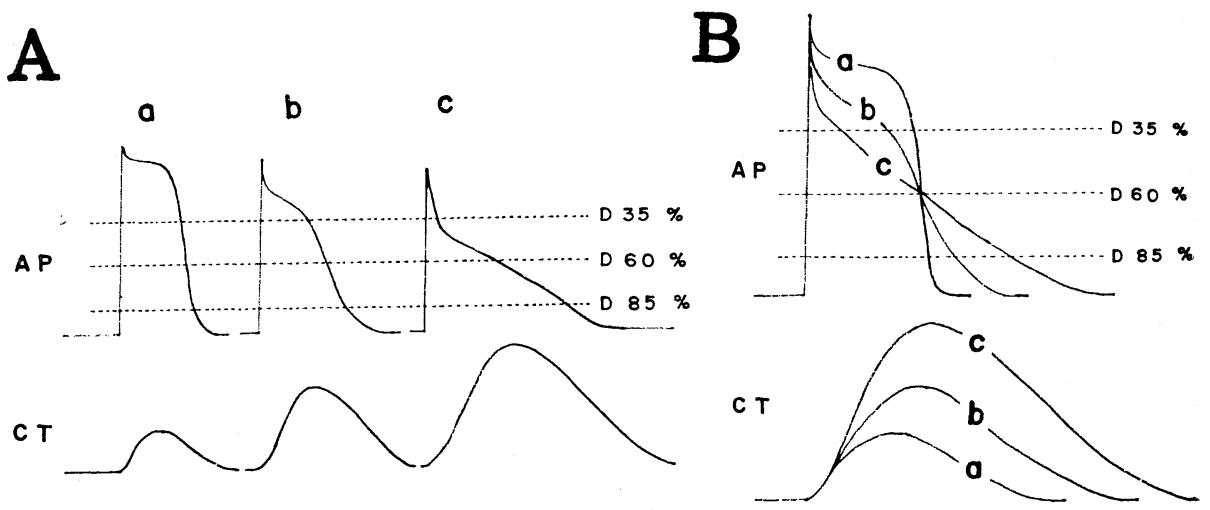

FIG. 12. Schematic representation illustrating the relationship between the shape of an action potential and the magnitude of the contractile force (A), and the superimposition of A (B). AP: Action potential. CT: Contractile tension. (a), (b) and (c) indicate small, moderate and large contraction respectively. Explanation is in text. 
constant in spite of marked changes in contractile force.

In other words, a change of the slope in the terminal portion of an action potential has the most intimate relation to a change in contraction strength: the steeper the slope of the terminal portion of repolarization the smaller was the contraction (FIG. 12).

On the other hand, about the basic relationship between action potential duration and contractile tension, no significant difference was recognized between the LSVC and LA (FIG. 11).

\section{DISCUSSION}

The existence of rhythmical contraction in the superior venae cavae (SVC), but the absence of it in the inferior vena cava (IVC) have been accounted for by microscopical findings. That is, the SVC near the heart have a collar of the striated muscle as well as vascular smooth muscle. In contrast, the IVC is virtually destitute of any striated muscle (OCHIAI, 1931 ; SENBA, 1941 ; Nishimoto, 1951). Thus, it was stated that contractions of the SVC played a role of a venous valve in preventing the regurgitation of the blood into the $\mathrm{SVC}$ from the right atrium (RA) at the atrial systole, for want of the venous valve at the vicinity of the SVC. On the other hand, the lack of contraction in the IVC was attributed to the existence of the venous valve (Eustachian valve) in its opening portion into the RA (OchIAI, 1931 ; SENBA, 1941).

The schema showing time delays in the spread of contraction (FIG. 1), which pointed out the orifice of the SVC and its neighbouring parts contracting $20 \sim 30 \mathrm{msec}$ earlier than the RA, is conceived to support the above-mentioned assumption. Namely, the contraction of the orifice of the SVC, by occurring its tightening for about $200 \mathrm{msec}$ or more (FIG. 2, A and B), act as a barrier to blood regurgitation into the SVC from the RA, which otherwise would be brought about by a subsequent atrial systole.

The contraction of the vena cava has been demonstrated also in man, by means of the endovenous angiocardiography, as a tightening of the mouth of the superior vena cava at the auricular systole (GARUSI, 1965).

From electron microscopic findings, KARRER (1959 and 1960) concluded that the musculature of small lung veins, thoracic portion of the inferior vena cava and other thoracic veins of a mouse was indeed of the cardiac type. Notwithstanding these many histological considerations, information about the physiological property of the venae cavae near the heart has been meagre.

It is of interest to speculate that the incomplete tetanus and the summation of contraction observed in some cases of the LSVC (FIG. 3, A-2) might explain the fact that the SVC exhibits the nature of the so-called "partial syncytia" similar to that of the smooth muscle (GoTO \& TAMAI, 1960). Every working unit of the muscle of the SVC seems unable to synchronize always 
with all others at rapid stimulation rates, due to the easily set up partial electrical conduction disturbance (FIG. 4), (MAshima et al. 1960).

On the other hand, considering that the LSVC, used in this experiment, was composed of two different musculatures, viz., the striated muscle of cardiac type and the vascular smooth muscle (OCHIAI, 1931), the somewhat tonic contraction demonstrated in FIG. 3, A-2 might be ascribable to the contraction of the vascular smooth muscle which connected with the striated muscle of cardiac type by means of such ephatic connection as observed at the transitional portion of the striated muscle and smooth muscle in the goldfish intestines (Yамамото, 1965).

Such conspicuous incomplete tetanus and summation as was seen in the LSVC (FIG. 3, A-2) could not be recorded from the LA, in spite of the rapid action potential firings of nearly the same frequency as in the LSVC. Therefore, the difference in the pattern of contraction between the LSVC and LA seems to be significant.

The phenomenon of multiple firings of the LSVC (FIG. 3, A-3) similar to that of the LA (FIG. 3, B-3) is thought to indicate that the SVC may have also such a "vulnerable period" as described in other heart muscles (BROOKS et al., 1955), and that in some peculiar conditions, e. g., application of aconitine (YANAGA et al.: Jap. Circul. J., in press.), a process similar to fibrillation or fibrillation itself can be induced in the vena caval muscle.

The dissociation of E-C coupling observed in the LSVC (FIG. 4) is conceived to be only an apparent effect, taking into consideration that an action potential was recorded from a single cell and contractile tension was obtained from a large amount of muscle fibers. In other words, because of severe and multiple electrical conduction blocks, and the excitation restricted within a narrow area close to the stimulus electrode, the numbers of excited muscle fibers are too small to represent an effective contractile tension which would normally be measurable by the strain gauge.

From the lower safety factor (FIG. 4) and the tetanus like contraction (FIG. 3, A-2), it may be assumed that the superior vena caval muscle proximal to the heart has to be ranked between the cardiac striated muscle with the property of complete syncytia, and the vascular smooth muscle with the property of partial syncytia, in view of intercellular functional connection (GoTo \& TAMAI, 1960).

Considering the drastic action of calcium ions $\left(\mathrm{Ca}^{++}\right)$on the contractility of cardiac muscle (Niedergerke, 1956 and 1959; WeIdmann, 1959), viz., the strength of contraction is controlled by the concentration of calcium in a superficially located region of the heart cell, it is reasonable to assume that the difference in contractile fashion between the LSVC and LA in the staircase phenomena (FIG. 5) may perhaps include the difference in $\mathrm{Ca}^{++}$-susceptibilty of their fiber membrane. Another possible explanation for the development 
of contractile inhibitory process in the normal staircase effect of the LA-strip (FIG. 5, B) are as follows: in comparison with the LSVC, (1) the LA is prone to reach a phase of "over load" (VAUGHAN WILliams, 1959) more easily at a rapid contraction; (2) the LA is more susceptible to "oxygen lack" (NIEDERGERKE, 1956); (3) the LA is more "exhausted" (MASHIMA et al., 1962) in the contractile system itself.

The difference of frequency producing the 'infinitesimal phase' in frequency-tension curve ( $\alpha$ and $\beta$ in FIG. 6) may be also ascribable to the difference in $\mathrm{Ca}^{++}$-sensitivity of the two.

A simple possibility to explain the decline in the magnitude of the rest contraction after a longer interval of the rest (200 sec or more) (FIG. 8) would be that $\mathrm{Ca}^{++}$has diffused out of a superficial layer of the cells into the Tyrode solution during the overly long quiescence, and that $\mathrm{Ca}^{++}$-deficiency of this layer give rise to a hypo-dynamic state. Hence, it can be said that the LSVC (solid line in FIG. 8) is prone to fall into hypo-dynamic state at a rather shorter quiescence than the LA (dotted line in FIG. 8).

Electrically induced arrhythmical pulsations of the atrio-caval preparation resulted in a conspicuous irregularity of the contraction strength and action potential configuration (FIG. 9). The mechanism to which the irregularity is ascribed seems somewhat similar to that of postextrasystolic or post-stimulation potentiation reported by other investigators (HoFFMAN et al., 1956; ROSIN \& FARAH, 1955 ; SIEBENS et al., 1959). In our experiment, however, the irregularity is more complicated, since the rhythmicity of the intrinsic pace maker (sinus node) itself falls into disorder. In spite of this, it must be emphasized that the magnitude of an arbitrary contraction ' $Z$ ' is well correlated with ' $y / x$ ' in the terminology illustrated in FIG. 10, C. This linear relationship between ' $z$ ' and ' $y / x$ ' is verified in both the LSVC and LA (FIG. 10 , A and $\mathrm{B})$. But the mechanism explicable of this relation between contraction strength and cycle length remains nearly obscure.

In this type of the arrhythmia (FIG. 9), the shape of the action potential in a large contraction (FIG. 12, A-c) resembled that of the SVC of a rabbit during perfusion with the $\mathrm{K}^{+}$-lack and/or $\mathrm{Ca}^{++}$-excess Tyrode solution; contrarily, the action potential in a small contraction (FIG. 12, A-a) was similar to that in the $\mathrm{K}^{+}$-excess and/or $\mathrm{Ca}^{++}$-lack situations (SAEKI et al.: unpublished data). These action potential changes seem virtually identical with those obtained from other cardial muscles in the $\mathrm{K}^{+}$-lack and/or $\mathrm{Ca}^{++}$-excess situations which caused a large contraction (BROoks et al., 1955; SuRAwICZ, 1959; WARE, 1961), and in the $\mathrm{K}^{+}$-excess and/or $\mathrm{Ca}^{++}$-lack, which resulted in a small contraction (HofFMAN \& Suckling, 1954; HofFMAN et al., 1956 ; HoFFMAN \& Kelly, 1959 ; Vaughan Williams, 1959).

Accordingly, it may be reasonable to assume that large contractions in these arrhythmias (FIG. 9) would be brought about by the instantaneous $\mathrm{Ca}^{++}$. 
excess at the superficial layer of muscle cells and the concurrent $\mathrm{K}^{+}$-lack at the immediate extracellular spaces. Small contractions, conversely, could be caused by the $\mathrm{Ca}^{++}$-lack and $\mathrm{K}^{+}$-excess.

Similarly, the action potential configuration with initial acceleration and terminal deceleration of the repolarization rate at the first rest contraction (FIG. 7, A-2 and B-2), might be attributable in part to the transient change of the external ionic compositions $\left(\mathrm{Ca}^{++}\right.$-excess and $\mathrm{K}^{+}$-lack $)$at the immediate extrafiber space.

\section{SUMMARY}

1. In the rabbit, there exist three venae cavae which empty into the right atrium (RA) by separate ostia: the left superior vena cave (LSVC), the right superior vena cava (RSVC), and the inferior vena cava (IVC). Electrical and mechanical responses of the venae cavae proximal to the heart were recorded and compared with those of the atria in vitro, by the aid of microelectrodes and a strain gauge transducer.

2. Spontaneous or electrically driven twitch tension, elicited by an single action potential, could be recorded from each of the superior venae cavae (SVC); however, neither action potential nor contraction was obtained from the IVC. This fact was discussed in relation to the histological findings.

3. The orifice of the SVC contracted $20 \sim 30 \mathrm{msec}$ earlier than the RA. This supports a hypothesis that rhythmical contractions of the SVC play a role of a venous valve in preventing blood regurgitation from the RA.

4. Under certain conditions, the LSVC exhibited a tetanus like contraction followed by the contractile summation, and a 'seeming dissociation' of E-C coupling. These results may suggest that the SVC have a more fragile intercellular connection than the atria, from the functional point of view.

5. Abrupt changes of stimulus frequency, from a higher $(4 \mathrm{cps})$ to a lower $(1 \mathrm{cps})$ value or vice versa, brought about normal or reverse staircase phenomena in the LSVC as well as in the left atrium (LA). In the LSVC, however, the contractile inhibitory process (fatigue) during normal staircase effect occurred more easily than in the LA.

6. Frequency-tension relationships in the LSVC and the LA were similar to each other on the whole. But the frequency causing the 'infinitesimal phase' of contractile force was significantly different from each other, e. g., the LSVC, $20 \pm 2.7 \mathrm{cps}$; the LA, $43 \pm 3.8 \mathrm{cps}$.

7. Phenomena of rest contraction were recognized in the LSVC as well as in the LA. The rest contraction reached a maximum after $60 \sim 200 \mathrm{sec}$ of quiescene in each of them. After the overly long rest-intervals ( $1000 \mathrm{sec}$ or more), however, the rest potentiation of the LSVC had disappeared more rapidly than that of the LA. That is, the LSVC falls into hypo-dynamic state more 
rapidly than the $\mathrm{LA}$.

The possible explanations for above-mentioned $(5,6$ and 7 ) differences between the LSVC and the LA were discussed with reference to the calciumsusceptibility of their fiber membranes.

8. In the electrically induced arrhythmias, an arbitrary contraction ' $z$ ' had, at first sight, no particular relation to the preceding cycle length ' $y$ ' nor the cycle length ' $\mathrm{x}$ ' prior to ' $\mathrm{y}$ '. However, highly positive correlation existed between ' $z$ ' and ' $y / x$ ' in each of the LSVC and LA. In these arrhythmias, moreover, the longer the $85 \%$-duration of an action potential and the shorter the $35 \%$-duration, the larger was the contraction; $60 \%$-duration was nearly constant regardless of the marked changes of twitch tension. The wide fluctuation of the strength of twitch tension was discussed with relation to calcium and/or potassium-concentration at the immediate extracellular spaces.

9. In general, the vascular muscle of the SVC proximal to the heart of a rabbit shows a similar nature to the atrial muscle in both electrical activity and contractility. Nevertheless, some difference in their nature was recognized. These differences may depend upon the complexity of the structure of the wall of the proximal SVC which is composed of striated muscle and smooth muscle, or the mixture of the two.

From the above-mentioned results, it seems reasonable to assume that the muscle of the superior venae cavae proximal to the heart has a position between the cardiac striated muscle and the vascular smooth muscle from the functional point of view. Further studies into this problem may be necessary.

\section{REFERENCES}

1) Arey, L. B. (1965). Developmental anatomy (Seventh ed.). W. B. Saunders Co., Philadelphia and London.

2) Brooks, C. McC., Hoffman, B. F., Suckling, E. E. And Orias, O. (1955). Excitability of the heart. Grunne \& Stratton, New York.

3) Brunton, T.L. And Fayrer, J. (1876). Note on independent pulsation of the pulmonary veins and vena cava. Proc. Roy. Soc., 25 : 174-176.

4) Cattel, Mck. And Gold, H. (1941). The relation of rhythm to the force of contraction of mammalian cardiac muscle. Am. J. Physiol., 133: 236-237.

5) Engelmann, T.W. (1897). Ueber dem Ursprung der Herzbewegungen und die physiologischen Einschaften der grossen Herzvenen des Forsches. Pfiugers Arch., $65: 109-214$.

6) Franklin, K. J. (1937). A monograph on veins. Charles C. Thomas, Springfield and Baltimore.

7) Garusi, G.F. (1965). Spiral movements of blood streams from the venae cavae into the right auricular appendage. Angiology, 15:488-495.

8) Goto, M. And Tamai, T. (1960). Modern aspect of the electrophysiology of involuntary muscle. Kinpodo Co., Tokyo (in Japanese).

9) Hajdu, S. (1953). Mechanism of staircase and contructure in ventricular muscle. Am. J. Physiol., $174:$ 371-380. 
10) Hoffman, B. F. And Suckling, E. E. (1954). Effect of heart rate on cardiac membrane potential and the unipolar electrogram. Am. J. Physiol., 179: 123-130.

11) Hoffman, B. F., Bindler, E. A. And Suckling, E. E. (1956). Postextrasystolic potentiation in cardiac muscles. Am. J. Physiol., 185: 95-102.

12) Hoffman, B. F., And Kelly, J. J. (1959). Effect of rate and rhythm of contraction of rat papillary muscle. Am. J. Physiol., $197: 1199-1204$.

13) Ito, M., Yanaga, T. And Mashiba, H. (1963). Studies on transmembrane potential and sino-caval conduction in mammalian cardiac and vascular smooth muscle. Kyushu J. Med., Sci. $14: 393-402$.

14) Ito, M., Yanaga, T., Saeki, K., Arita, M., Ishihara, M. And Mashiba, H. (1964). Studies on sino-caval conduction of the rabbit with microelectrodes. Jap. J. Physiol., 14 : 439-449.

15) Karrer, H. E. (1959). The striated musculature of blood vessels. I. General cell morphology. J. Biophysic. and Biochem. Cytol., 6 : 383-403.

16) Karrer, H. E. ANd Cox, J. (1960). The striated musculature of blood vessels. II. Cell interconnection anc cell surface. J. Biophysic. and Biochem. Cytol., 8 : 135-150.

17) Mashiba, H. Yanaga, T. And Ito, M. (1963). Studies on transmembrane action potential of mammalian vascular smooth muscle and myo-myo-excitation transmission between cardiac and vascular muscle. Kyushu J. Med. Sci., 14 : 121-130.

18) Mashima, H., Matsumura, M. And Nakayama, Y. (1962). On the coupling relation between potential and mechanical response during repetitive stimulation in frog sartorius muscle. Jap. J. Physiol., 12: 324-336.

19) Mashima, H. (1965). The factors influencing the magnitude of contraction. Igaku no Ayumi, 54 : 385-391 (in Japanese).

20) Niedergerke, R. (1956). The staircase phenomenon and the action of calcium in the heart. J. Physiol., $134: 569-583$.

21) Niedergerke, R. (1959). Calcium and the activation of contraction. Experientia, $15: 128-130$.

22) Nishimoto, K. (1951). A study of automatic contractility of the vena cava proxinal to the heart. Hiroshima Igaku, $4: 643-646$ (in Japanese).

23) Ochial, K. (1931). Histological studies on mammalian thoracic veins, with special reference to striated muscle fibers in the wall of veins. Aichi Igaku Zasshi, 38 : 843-896 (in Japanese).

24) Rosin, H. ANd FArah, A. (1955). Post-stimulation potentiation of contractility in the isolated auricle of the rabbit. Am. J. Physiol., $180: 75-82$.

25) SENBA, T. (1941). A study of automatic contractility in the vena cava proximal to the heart. Nippon Seirigaku Zasshi, 5:370-378 (in Japanese).

26) Siebens, A. A., Hoffman, B. F., Cranefield, P. F., And Brooks, C. McC. (1959). Regulation of contractile force during venticular arrhythmias. Am.J. Physiol., 197: 971-977.

27) Sleator, W., Furchgott, R. F., Gubareff, T. D. And Krespi, V. (1964). Action potentials of guinea pig atria under conditions which alter contraction. Am.J. Physiol., 206 : 270-282.

28) Surawicz, B., Lepeschikin, E., Herrlich, H. C. and Hoffman, B. F. (1959). Effect of potassium and calcium deficiency on the monophasic action potential, electrogramm and contractility of isolated rabbit hearts. Am. J. Physiol., 196: 1302-1307.

29) Vane, J.R. (1957). Frequency of contraction as a factor in the action of drugs on isolated heart tissue. J. Physiol., 138: 16-17.

30) Vaughan Williams, E. M. (1959). A study of intracellular potentials and contractions in atria, including evidence for an after-potential. J. Physiol. 149: 78-92. 
31) WARE, F. (1961). Effect of calcium deficiency and excess on transmembrane potentials in frog heart. Am. J. Physiol., 201: 1113-1119.

32) Weidmann, S. (1959). Effect of increasing the calcium concentration during a single heart-beat. Experientia, 15:128-128.

33) Yамамото, T. (1965). Some aspects of transitional portion between the smooth muscle and striated muscle, including cellular connection and neuro-muscular junction. Igaku no Ayumi, $54: 32-36$ (in Japanese). 